\title{
Açóes para 0 enfrentamento da violência contra a mulher em duas unidades de atenção primária à saúde no município do Rio de Janeiro*
}

\author{
Tatiana dos Santos Borsoi ${ }^{1}$ \\ Elaine Reis Brandão² \\ Maria de Lourdes Tavares Cavalcanti ${ }^{3}$
}

BORSOI, T.S.; BRANDÃO, E.R.; CAVALCANTI, M.L.T. Actions addressing violence against women at two primary healthcare centers in the municipality of Rio de Janeiro.

Interface - Comunic., Saúde, Educ., v.13, n.28, p.165-74, jan./mar. 2009.

The aim here was to investigate and discuss actions for addressing domestic violence within the scope of the Women's Comprehensive Healthcare Program. For this, it was sought to survey and compare professional practices at two health centers in the municipality of Rio de Janeiro, of which one was a referral center caring for victims of violence. Eight semistructured in-depth interviews were conducted with professionals from the teams of the Women's Comprehensive Healthcare Program. Although both centers often identified users who had been victims of domestic violence, the professionals at the referral center were shown to be better prepared for identifying this problem when it was not explicitly presented, and for developing welcoming practices within their own healthcare unit. What differentiated the two units was how the problem was addressed, which was related to the way in which each service understood violence as a demand inherent to the healthcare sphere.

Key words: Primary healthcare. Women's health. Violence against women. Domestic violence. Gender violence.
Propôs-se conhecer e discutir ações voltadas para o enfrentamento da violência doméstica no âmbito do Programa de Assistência Integral à Saúde da Mulher. Para tanto, buscou-se levantar e comparar as práticas profissionais em duas unidades de saúde do município do Rio de Janeiro, sendo uma delas referência para $\mathrm{o}$ atendimento às vítimas de violência. Foram realizadas oito entrevistas em profundidade, semiestruturadas, com profissionais das equipes do Programa de Saúde da Mulher. Embora ambas as unidades identifiquem frequentemente usuárias vítimas de violência doméstica, na unidade de referência os profissionais se mostraram mais preparados para identificar o problema quando este não se apresenta de forma explícita e para desenvolver ações de acolhimento dentro da própria unidade de saúde. O que diferencia as duas unidades é o encaminhamento dado ao problema, relacionado com a forma como cada serviço entende a violência como demanda inerente também à esfera da saúde.

Palavras-chave: Atenção primária à saúde. Saúde da mulher. Violência contra a mulher. Violência doméstica. Violência de gênero.

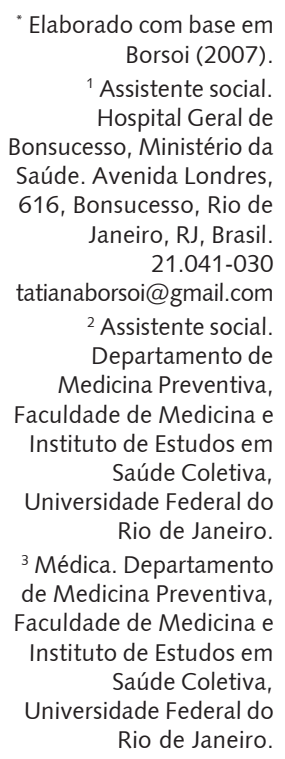
Borsoi (2007).

${ }^{1}$ Assistente social. Hospital Geral de Bonsucesso, Ministério da Saúde. Avenida Londres, 616, Bonsucesso, Rio de Janeiro, RJ, Brasil. 21.041-030

tatianaborsoi@gmail.com ${ }^{2}$ Assistente social. Departamento de Medicina Preventiva, Faculdade de Medicina e Instituto de Estudos em Saúde Coletiva, Universidade Federal do Rio de Janeiro.

${ }^{3}$ Médica. Departamento de Medicina Preventiva, Faculdade de Medicina e Instituto de Estudos em Saúde Coletiva,

Universidade Federal do Rio de Janeiro. 
AÇÕES PARA O ENFRENTAMENTO DA VIOLÊNCIA ..

\section{Introdução}

Nas últimas décadas, a violência contra a mulher tem sido reconhecida como um problema de Saúde Pública, por organismos internacionais como a Organização Mundial de Saúde (OMS) e a Organização Pan-Americana de Saúde (Opas). A OMS relaciona essa violência à maior ocorrência de diversos problemas de saúde física, reprodutiva e mental, assim como ao maior uso de serviços de saúde por parte das mulheres (Schraiber, 2001).

A expressão "violência contra a mulher" inclui as mais diversas situações: violência física, sexual e psicológica, cometida por parceiro íntimo; estupro; abuso sexual de meninas; assédio sexual no local de trabalho; violência étnica e racial; violência cometida pelo Estado por ação ou omissão; mutilação genital; violência e assassinatos ligados ao dote; estupro em massa nas guerras e conflitos armados (OEA, 1996).

Embora a violência possa ser cometida por diversos perpetradores, estudos populacionais e em serviços de saúde indicam que há um maior risco de agressão às mulheres por parte de parceiros íntimos do que por estranhos (Schraiber et al., 2002; Deslandes, Gomes, Silva, 2000; Heise, Pitanguy, Germain, 1994).

Segundo estudo internacional realizado por Heise, Pitanguy e Germain (1994), a violência doméstica atinge cerca de $20 \%$ a $50 \%$ das mulheres ao redor do mundo ao menos uma vez em toda a vida.

No Brasil, as pesquisas realizadas com dados populacionais (Fundação IBGE, 1990), em delegacias especiais de atendimento à mulher (Brandão, 2006, 1997; Soares, 1999), ou em serviços de saúde (Schraiber et al., 2002; Deslandes, Gomes, Silva, 2000), apontam também um padrão centrado na violência doméstica, com o parceiro ou ex-parceiro como agressor. Os episódios violentos são repetitivos e tendem a se tornar mais graves caso não haja uma ação que os interrompa.

Nos últimos anos, foram criados, no Brasil, serviços voltados para mulheres em situação de violência, tais como: as delegacias de defesa da mulher, as casas-abrigo e os centros de referência multiprofissionais (Schraiber et al., 2002).

No setor saúde, a questão passa a ter importância com a implantação do Programa de Assistência Integral à Saúde da Mulher (Paism) na década de 1980, com a proposta de explorar questões de gênero e abordar as necessidades integrais de saúde da mulher (Costa, 1999; Schraiber, D'Oliveira, 1999). Entretanto, esta iniciativa não significou, na época, mudanças expressivas na atenção à mulher em situação de violência, por parte dos serviços de saúde, uma vez que, na prática, as ações priorizadas se restringiram à saúde reprodutiva (Correa, Piola, 2003).

No Brasil, a preocupação em capacitar os profissionais para identificarem a presença de mulheres em situação de violência nos serviços de saúde se inicia no final da década de 1990.

Atualmente, o Ministério da Saúde e diversas organizações não-governamentais feministas têm produzido material didático, com orientações sobre o tema, e oferecido treinamentos aos profissionais de saúde de modo que eles possam identificar, apoiar e dar o devido encaminhamento às vítimas de violência. Um avanço já pode ser percebido no que tange ao enfrentamento da violência sexual pelos serviços de saúde. Tais medidas resultam tanto da compreensão de que a violência representa uma violação dos direitos humanos, como também do reconhecimento de que esta é uma importante causa do sofrimento e adoecimento, sendo fator de risco para diversos problemas de saúde (físicos e psicológicos).

Apesar desses avanços, os serviços de saúde nem sempre oferecem uma resposta satisfatória para o problema, que acaba diluído entre outros agravos, sem que seja levada em consideração a recorrência do ato que ocasionou aquela morbidade.

Segundo Silva (2003), esta "invisibilidade" da violência decorre do fato de alguns setores ainda se limitarem a cuidar dos sintomas das doenças e não contarem com instrumentos capazes de identificar o problema. Desta forma, as intervenções acabam por mostrar respostas insuficientes dos serviços para as necessidades das mulheres, pois, uma vez que a situação de violência não se extingue, as repercussões sobre o adoecimento físico ou mental ressurgem e voltam a pressionar os serviços (Schraiber et al., 2002). 
${ }^{4}$ Técnica em que o profissional de saúde busca identificar sinais de doenças (no caso, de violência doméstica) em pacientes, independente de qualquer demanda explícita.
Cavalcanti (2002) e Tuesta (1997), no entanto, constataram que os profissionais de saúde não têm dificuldades em identificar a violência, uma vez que suspeitam frequentemente desta. A maior dificuldade dos profissionais, segundo estas autoras, está em não saber como encaminhar a questão.

A proposta para os serviços de saúde tem sido, simplesmente, detectar a violência com a "busca ativa" ${ }^{4}$ de rotina. Porém, quando detectada a violência, a demanda é rejeitada como "não-doença", porque social, ou ao contrário, é percebida como patologia, reduzindo ao corpo individual algo que é fruto das interações humanas. Schraiber (2001) chama atenção que, ao se aplicar a idéia de doença à violência, pode-se incorrer no aprofundamento da idéia de vitimização, colocando as mulheres nesta situação, assim como os doentes, como sujeitos incapazes que necessitam de tutela especializada.

Por estarem próximos à maioria das mulheres, que, por um motivo ou outro, os utilizam em seu cotidiano, os serviços de saúde têm o dever de se constituírem como um local de acolhimento e elaboração de projetos de apoio, ao invés de serem mais um obstáculo na tentativa empreendida pelas mulheres de transformar sua situação de opressão.

A alta prevalência do problema nos serviços de saúde foi constatada por estudos tanto nacionais (Silva, 2003; Schraiber et al., 2002; Deslandes, Gomes, Silva, 2000), como internacionais (Mccauley et al., 1995; American Medical Association, 1994). No entanto, depois de constatada a presença de mulheres em situação de violência como demanda recorrente no setor saúde, é preciso refletir sobre como o setor tem absorvido e encaminhado o problema.

As unidades de saúde são importantes na detecção da violência doméstica, porém sua identificação deve se constituir no início de um processo que busque apoiar tais usuárias na superação do problema.

Nesse sentido, realizou-se uma investigação em duas unidades de atenção primária à saúde do município do Rio de Janeiro, em 2006, com o objetivo de conhecer como tais serviços absorvem e encaminham as demandas relativas às situações de violência percebidas pelos profissionais de saúde no atendimento cotidiano às usuárias.

O enfoque na atenção primária se justifica por ser este um local privilegiado para o desenvolvimento de ações de prevenção, reflexão e orientação sobre o tema, pois tem uma grande cobertura e possibilita um contato mais estreito com as mulheres, podendo reconhecer e acolher o caso antes de incidentes mais graves.

\section{Metodologia}

Optou-se pela metodologia de natureza qualitativa, uma vez que a pesquisa tem caráter exploratório e destina-se a entender o fenômeno, segundo a perspectiva dos profissionais de saúde envolvidos.

O material empírico é composto por observação de campo e entrevistas em profundidade com oito profissionais de saúde do Paism, de duas unidades de atenção primária do município do Rio de Janeiro, ambas localizadas na zona norte da cidade. As duas unidades são referências para as ações do Paism, sendo que uma é referência também para o atendimento às vitimas de violência em sua área programática.

Em cada unidade estudada, foi entrevistado um representante das seguintes categorias profissionais, a saber: médico, enfermeiro, assistente social e psicólogo. A princípio, a pesquisa procurava entrevistar todos os profissionais do Paism de 
cada unidade. No entanto, na unidade que não é referência para o atendimento às vítimas de violência, três profissionais não foram incluídos (duas assistentes sociais e uma psicóloga), por se recusarem a participar. As entrevistas foram realizadas no próprio serviço de saúde, com duração média de sessenta minutos. A observação de campo foi realizada pelo período de um mês em cada unidade, nos grupos de planejamento familiar e pré-natal.

O material empírico é parte de uma pesquisa para dissertação de mestrado sobre as ações para o enfrentamento da violência doméstica no âmbito do Paism. Para este artigo, foram selecionados alguns eixos do roteiro de entrevista para serem abordados: 1) apreender como os serviços se estruturam para absorver a questão da mulher em situação de violência; 2) como esta demanda emerge nos serviços estudados; 3 ) conhecer as ações realizadas dentro das unidades para o enfrentamento dos casos de violência contra a mulher em âmbito doméstico; 4) entender a percepção dos profissionais de saúde sobre suas práticas e os limites enfrentados; 5) comparar as ações das duas unidades.

Para fins de análise, as unidades estudadas serão designadas como: unidade referência e unidade não-referência, distinguindo-se, assim, a unidade já treinada no tema da violência de gênero.

É importante ressaltar que a ética da pesquisa considerou: 1) o consentimento informado dos profissionais entrevistados; 2) a explicitação, aos entrevistados, sobre seu direito de interromper a entrevista a qualquer momento; 3) a garantia do sigilo dos dados coletados; 4) a garantia de retorno dos resultados após a conclusão da pesquisa.

O projeto foi aprovado pelo Comitê de Ética em Pesquisa da Secretaria Municipal de Saúde do Rio de Janeiro.

\section{Resultados e discussão}

Os profissionais entrevistados têm de 32 a cinquenta anos. Foram sete mulheres e apenas um homem, todos com nível superior e do quadro permanente de profissionais da unidade. Os entrevistados conformam um grupo com vasta experiência em atenção à saúde da mulher, o que foi constatado pelo longo tempo de serviço nas unidades estudadas (de três a vinte anos); experiências anteriores na assistência à saúde da mulher; e pela participação em cursos e capacitações sobre o assunto.

No que diz respeito à violência contra a mulher, os profissionais da unidade referência passaram por uma capacitação para o atendimento às vitimas, e a unidade mantém a continuidade da discussão sobre o assunto em seu centro de estudos. Na unidade não-referência, apenas a assistente social passou por uma especialização em violência doméstica.

As ações do Paism realizadas nas duas unidades estudadas se dividem em: atendimentos individuais e grupos educativos com gestantes e usuárias do planejamento familiar. O espaço de grupo é reconhecido, pelos profissionais das duas unidades estudadas, como lócus privilegiado para a prevenção e identificação de casos de violência doméstica ou de gênero.

Entretanto, nas ações de assistência à saúde da mulher nas duas unidades ${ }^{5}$, a violência doméstica é trabalhada apenas no grupo de planejamento familiar. Os profissionais apontam este espaço como um lugar estratégico para se trabalhar a prevenção da violência de gênero, uma vez que o programa cria um vinculo entre a usuária e a instituição, pela possibilidade de um atendimento continuado.

Os profissionais relatam identificar, com frequência, casos de mulheres que

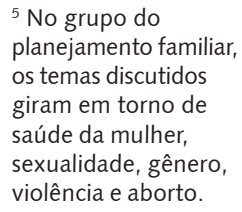

${ }^{5}$ No grupo do planejamento familiar, os temas discutidos giram em torno de saúde da mulher, sexualidade, gênero, violência e aborto. 
${ }^{6}$ Os assuntos abordados são: prénatal, cuidado com o recém-nascido, maternidade $\mathrm{e}$ amamentação. sofrem violência, no decorrer da atividade de grupo, especialmente quando o tema é trazido para debate - momento em que algumas mulheres choram ou relatam as agressões sofridas na esfera conjugal.

No que se refere ao grupo de gestantes, ele foi apontado apenas pelos profissionais da unidade referência, como um lugar onde a prevenção da violência doméstica é trabalhada. Nessa unidade, os temas violência e gênero são abordados durante os encontros de gestantes, e os profissionais relatam já terem identificado, nesses grupos, gestantes que viviam situações de violência. Na unidade nãoreferência, este tema não está presente nas discussões do grupo de gestantes ${ }^{6}$. Percebe-se que há uma "sacralização" do período gestacional, que pode ser notado, inclusive, no depoimento de uma profissional entrevistada, que justifica a não discussão do tema violência com gestantes por elas estarem vivendo "um outro momento em suas vidas".

É importante notar que a literatura da área aponta uma alta incidência de violência cometida contra gestantes. Destacamos o estudo realizado por Schraiber et al. (2002) em uma unidade primária de saúde em São Paulo, onde 21,3\% das gestantes atendidas no período pesquisado relataram sofrer agressões por parte do companheiro.

O discurso enaltecedor da maternidade não encontra respaldo na realidade. $\mathrm{O}$ suplemento especial sobre violência contra a mulher do Maternal and Child Health Journal (Spitz, Marks, 2000) constatou que a violência pode ser um problema mais comum entre mulheres grávidas do que a pré-eclampsia e a diabetes gestacional. Estes dados apontam a urgência de se organizar os serviços de saúde de forma a possibilitar a identificação do problema na assistência pré-natal.

\section{O reconhecimento da violência como uma demanda para o serviço de saúde}

Em ambos os serviços estudados, pode-se constatar, por meio dos relatos dos profissionais de saúde e da observação realizada, que a demanda da mulher em situação de violência se apresenta sempre de forma implícita, ou seja, não é esta situação que diretamente as leva às unidades de saúde.

A violência aparece como demanda "explícita" apenas nos casos de violência sexual praticada por estranhos. A violência praticada contra as mulheres pelos parceiros, no âmbito doméstico, seja ela física, sexual, ou psicológica, não se constitui uma demanda imediata para os serviços. Todos os casos trabalhados são detectados pelos profissionais, seja no atendimento individual ou nos grupos, ocorrendo com frequência.

No entanto, é preciso fazer uma diferenciação entre as duas unidades estudadas: quando perguntados se a violência contra a mulher era uma demanda frequente ao serviço, os profissionais da unidade não-referência respondiam que a mesma não aparecia no serviço. Porém, no transcorrer da entrevista, percebia-se que, na realidade, a violência nunca se apresentava como uma demanda imediata ao serviço, sendo expressiva como demanda "implícita", em especial na prática clínica dos médicos, onde boa parte das queixas das mulheres estava relacionada com a violência doméstica, como mostra o relato da médica entrevistada:

[...] geralmente [a usuária] não vem com essa queixa [de violência] assim [...] geralmente elas chegam com queixa ginecológica, é quando então aparecem as violências, é algo que não é de pequena intensidade não, é de muita intensidade [...] mulheres que vêm aqui, muitas delas são maltratadas por seus parceiros [...] Mais ou menos 40\% [...] essa 
estatística, é se o profissional de saúde ver e perguntar [...] porque as vezes elas se fecham mesmo $[\ldots]$.

Na unidade referência, ao contrário, a demanda implícita é prontamente identificada como algo que compete ao serviço de saúde. Quando perguntados sobre a frequência com que a violência se apresenta como demanda na unidade, eles se referem a essa violência que é percebida no atendimento cotidiano às usuárias.

Esta constatação sugere que, apesar de conseguirem identificá-la, alguns profissionais ainda encontram dificuldade em perceber a violência como demanda de ação específica para o setor saúde, o que, certamente, tem repercussões no seu envolvimento e intervenção posterior.

\section{Encaminhamentos}

A demanda identificada é prontamente acolhida, seja na clínica médica ou nos grupos educativos das duas unidades estudadas. Há, no entanto, uma diferenciação quanto ao seu encaminhamento.

Na unidade não-referência, os profissionais, ao acolherem o problema, oferecem todas as informações sobre os direitos da mulher no que tange à denúncia policial e às redes de suporte que podem ser acionadas nesses casos. Indagam ainda sobre a possibilidade e a vontade de a mulher romper com a situação violenta. No entanto, a abordagem se extingue, tendo dois desdobramentos possíveis: primeiro, caso aceite a possibilidade de denunciar à polícia, a mulher é encaminhada "para fora" da unidade (delegacias, abrigos, etc...), e os profissionais desconhecem o que acontece deste momento em diante. Não há um acompanhamento por parte dos profissionais da equipe. Segundo, se a mulher não aceita as alternativas apresentadas, os profissionais se desincumbem de fazer algo - "largam de mão", alegando não poder "obrigá-la a denunciar".

Na unidade referência, além de acolherem tal demanda e informarem sobre redes de apoio, os profissionais acompanham o caso por longo tempo. Eles se referem aos encaminhamentos internos à instituição e ao trabalho em equipe. Há uma tentativa de envolver os diversos profissionais no atendimento, para que essa mulher seja acompanhada na própria unidade, e não precise peregrinar por outros serviços.

Ainda que a mulher não queira denunciar o companheiro à polícia, a equipe se envolve no caso e os profissionais marcam atendimentos continuados para garantir sua volta à unidade. A equipe tenta, ainda, incluir outros familiares no atendimento, como filhos adultos, ou parentes próximos que possam servir de rede social de apoio para essa mulher.

Outra estratégia utilizada pela equipe é a inclusão do agressor na proposta de atendimento prestado. Tentam chamá-lo para conversar e inseri-lo no atendimento em curso. Porém, segundo os entrevistados, esta estratégia é a mais difícil de ser colocada em prática, não apenas pela resistência do homem em acatar o convite, mas também pelo risco que o próprio profissional de saúde corre.

A equipe busca trabalhar a dimensão afetiva (com intervenção da psicóloga) e a inserção ocupacional (com a assistente social) junto à usuária vítima de violência, e tenta não "oferecer" alternativas, mas "elaborá-las" em conjunto com a mesma.

Pode-se perceber então que, enquanto na unidade não-referência há o encaminhamento "para fora" da mesma e posterior "perda" do controle sobre os desdobramentos de sua ação, na unidade referência, a estratégia destina-se a envolver a equipe no acompanhamento das ações subsequentes.

Acredita-se que as práticas profissionais estão relacionadas com o modo como a violência é entendida pelos entrevistados. Intervir sobre um fenômeno requer compreendê-lo como uma demanda para sua ação. Constatamos que, acostumados a trabalhar com a demanda espontânea, onde os indivíduos buscam os serviços a partir dos seus sintomas, os profissionais da unidade não-referência ainda apresentam dificuldades em conceber a violência doméstica como uma demanda inerente a sua prática profissional no setor saúde. 


\section{Percepção sobre as práticas}

Os profissionais das duas unidades percebem que o trabalho com a violência não depende exclusivamente do setor saúde, sendo necessário o suporte de uma rede intersetorial (polícia, justiça, assistência social, educação etc.).

No depoimento dos profissionais, fica clara a preocupação para que o trabalho realizado não cause mais danos à mulher, além dos que ela apresenta ao serviço. O enfrentamento da violência é algo que ultrapassa o serviço de saúde, envolvendo a opção e o protagonismo da mulher, assim como a ação dos mais diversos setores.

Os profissionais das duas unidades percebem suas práticas como permeadas por "limites", tais como: capacidade da própria mulher em conseguir sair ou não da situação violenta; e a "ineficácia" da rede de atendimento intersetorial.

Embora estas limitações tenham sido apontadas pelos profissionais das duas unidades, o que os diferencia é a relação que estabelecem com estes limites. Enquanto na unidade não-referência os profissionais dizem preferir "não se envolver", por anteverem os obstáculos e não poderem garantir, à mulher, a resolução do seu problema, na unidade referência, os profissionais acolhem tal demanda. Entretanto, constatam que, embora tentem realizar um bom atendimento, muitas vezes, não conseguem impedir que a mulher seja "revitimizada", como mostra o relato da assistente social:

[...] eu tive uma usuária [...] na época que eu identifiquei, ela foi pra delegacia de mulheres, fez a ocorrência e teve lá um acompanhamento psicológico, jurídico[...] eles a colocaram em um abrigo, mas depois ela veio embora, porque ela perdeu tudo, o pouquinho que ela levou pra lá [...] ela disse que tinha um fogão que tinha comprado com tanto sacrifício, um berço, uma cama, que roubaram tudo dela [...] aí ela voltou a morar com ele [...] e assim, até hoje ela continua nessa situação [...].

Como se pode observar no relato, há uma limitação que ultrapassa o setor saúde. Além de ser numericamente reduzida, a rede de apoio social às mulheres em situação de violência é precária e acaba por não garantir plenamente a proteção das usuárias.

O setor saúde pode intervir na questão da violência contra a mulher, ao identificar o problema, acolher e apoiar as usuárias, acompanhando estes casos ao longo do tempo, no entanto, a resolução do problema não Ihe compete exclusivamente. É fundamental que a rede intersetorial se organize de forma a garantir que as mulheres não tenham seus direitos de cidadania usurpados.

\section{Conclusão}

Observa-se que a violência doméstica é uma realidade bastante comum entre as usuárias dos serviços de saúde estudados, apesar de não se apresentar como a queixa principal que as leva a procurar atendimento.

Este estudo aponta, no entanto, que apesar da importância da identificação, hoje se coloca uma nova necessidade: refletir sobre o encaminhamento dado aos casos já detectados, e instrumentalizar os profissionais para o enfrentamento do problema.

Como pode ser observado, as unidades estudadas apresentam-se como um "terreno fértil" para a ampliação das ações de prevenção à violência contra a mulher, uma vez que esta é uma realidade comum entre suas usuárias e frequentemente identificada pelos profissionais entrevistados.

A maior dificuldade parece residir nas ações posteriores à identificação e ao primeiro acolhimento. A pesquisa mostrou que o treinamento realizado foi um importante diferencial no atendimento prestado às mulheres em situação de violência. Os profissionais da unidade referência estão mais dispostos a incorporar, em suas práticas, os casos de violência que não se mostram como demanda direta ao serviço, e a prestar um atendimento continuado na própria unidade. 
Na unidade não-referência, ao contrário, os profissionais valorizam mais a identificação dos casos de violência e o encaminhamento aos serviços especializados.

A ênfase apenas na identificação dos casos de violência pode acabar por ter um efeito diverso do esperado, uma vez que, identificada a violência, a demanda pode ser desqualificada como algo que não requer uma intervenção também do setor saúde (Schraiber, 2001). Considerar as questões relativas à violência nas práticas de saúde é tomar os problemas relativos aos serviços como concernentes ao sujeito social (Schraiber, D'Oliveira, 1999).

É necessário compreender que o atendimento na unidade de saúde não pode se resumir a uma prática medicalizadora. É imprescindível que haja o compromisso de se considerarem os problemas sociais que atravessam a vida dos usuários do serviço como relacionados ao processo de adoecer.

Embora este estudo tenha mostrado a validade do treinamento realizado na unidade referência - o que desencadeou um melhor acolhimento dos casos -, ele também aponta a necessidade de se fortalecerem todas as instituições envolvidas no enfrentamento da violência, uma vez que, sem essa articulação intersetorial, a mulher acaba por ser novamente vitimizada, ao não ter seus direitos garantidos.

O presente estudo foi desenhado para fornecer dados sobre as ações voltadas para o enfrentamento da violência doméstica na assistência primária à saúde, e se configura como pesquisa em serviço de saúde.

\section{Colaboradores}

Os três autores trabalharam no projeto da pesquisa. Tatiana Borsoi realizou a revisão bibliográfica, o trabalho de campo, a análise dos dados e redigiu o artigo, sob orientação de Elaine Reis Brandão e Maria de Lourdes T. Cavalcanti. A revisão final do artigo foi feita por Elaine Brandão.

\section{Referências}

AMERICAN MEDICAL ASSOCIATION. Diagnostic and treatment guidelines on domestic violence. Chicago: AMA, 1994.

BRANDÃO, E.R. Renunciantes de direitos? A problemática do enfrentamento público da violência contra a mulher: o caso da Delegacia da Mulher. Physis, v.16, n.2, p.207-31, 2006.

Nos corredores de uma Delegacia da Mulher: um estudo etnográfico sobre as mulheres e a violência conjugal. 1997. Dissertação (Mestrado) - Instituto de Medicina Social, Universidade do Estado do Rio de Janeiro, Rio de Janeiro. 1997.

CAVALCANTI, M.L.T. A abordagem da violência intrafamiliar no Programa Médico de Família: dificuldades e potencialidades. 2002. Tese (Doutorado) - Instituto Fernandes Figueira, Fundação Oswaldo Cruz, Rio de Janeiro. 2002.

CORREA, S.O.; PIOLA, S.F. Balanço 1998-2002: aspectos estratégicos, programáticos e financeiros. Brasília: Ministério da Saúde, 2003.

COSTA, A.M. Desenvolvimento e implementação do PAISM no Brasil. In: GIFFIN, K.; COSTA, S.H. (Orgs.). Questões da saúde reprodutiva. Rio de Janeiro: Editora Fiocruz, 1999. p.319-35. 
DESLANDES, S.F.; GOMES, R.; SILVA, C.M.F.P. Caracterização dos casos de violência doméstica contra a mulher atendidos em dois hospitais públicos do Rio de Janeiro. Cad. Saude Publica, v.16, n.1, p.129-37, 2000.

FUNDAÇÃO IBGE. Participação político-social: justiça e vitimização. Rio de Janeiro: IBGE, 1990.

HEISE, L.; PITANGUY, J.; GERMAIN, A. Violence against women: the hidden health burden. World Bank Discussion Papers, n.255, 1994. Disponível em: <http://wwwwds.worldbank.org >. Acesso em: 05 jan. 2007.

MCCAULEY, J. et al. The "Battering Syndrome": prevalence and clinical characteristics of domestic violence in primary care internal medicine practices. Ann. Intern. Med., n.123, p.737-46, 1995.

OEA. Convenção Interamericana para prevenir, punir e erradicar a violência contra a mulher: convenção de Belém do Pará, Brasil. São Paulo: Comitê Latino Americano e do Caribe para Defesa dos Direitos da Mulher (CLADEM)/IPÊ, 1996.

OLIVEIRA, E.A. A violência contra mulheres: demandas espontâneas e busca ativa em Unidades Básicas de Saúde. Saude Soc., n.9, sup.1/2, p.3-15, 2000.

OLIVEIRA, E.M. et al. Atendimento às mulheres vítimas de violência sexual: um estudo qualitativo. Rev. Saude Publica, v.39, sup.3, p.376-82, 2005.

SCHRAIBER, L.B. Violência contra mulheres e políticas de saúde no Brasil: o que podem fazer os serviços de saúde? Rev. USP, v.51, p.104-13, 2001.

SCHRAIBER, L.B.; D'OLIVEIRA, A.F.P.L. Violência contra a mulher: interfaces com a saúde. Interface - Comunic., Saude, Educ., v.3, n.5, p.11-27, 1999.

SCHRAIBER, L.B. et al. Violência contra a mulher: estudo em uma unidade de atenção primária à saúde. Rev. Saude Publica, v.36, n.4, p.470-7, 2002.

SILVA, I.V. Violência contra mulheres: a experiência de usuárias de um serviço de urgência e emergência de Salvador, Bahia, Brasil. Cad. Saude Coletiva, v.19, sup.2, p.263-72, 2003.

SOARES, B. Mulheres invisíveis: violência conjugal e novas políticas de segurança. Rio de Janeiro: Civilização Brasileira, 1999.

SPITZ, A.M.; MARKS, J.S. Violence and reproductive health. Mater. Child Health J., v.4, n.2, p.77-8, 2000.

TUESTA, A J.A. Gênero e violência no âmbito doméstico: a perspectiva dos profissionais de saúde. 1997. Dissertação (Mestrado) - Escola Nacional de Saúde Pública, Fundação Oswaldo Cruz, Rio de Janeiro. 1997. 
BORSOI, T.S.; BRANDÃO, E.R.; CAVALCANTI, M.L.T. Acciones para afrontar la violencia contra la mujer en dos unidades de atención primaria a la salud en el municipio de Rio de Janeiro, Brasil. Interface - Comunic., Saúde, Educ., v.13, n.28, p.165-74, jan./mar. 2009.

La propuesta ha sido conocer y discutir acciones destinadas a afrontar la violencia doméstica en el àmbito del Programa de Asistencia Integral a la Salud de la Mujer. Para tanto se ha tratado de levantar y comparar las prácticas profesionales en dos unidades de salud del municipio de Rio de Janeiro, siendo una de ellas referencia para el atendimiento a las víctimas de violencia. Se han realizado ocho entrevistas en profundidad, semi-estructuradas, con profesionales de los equipos del Programa de Salud de la Mujer. Aunque ambas unidades identifiquen frecuentemente usuarias víctimas de violencia doméstica, en la unidad de referencia los profesionales se muestran más preparados para identificar el problema cuando no se presenta de forma explícita y para desarrollar las acciones de acogimiento dentro de la propia unidad de salud. Lo que diferencía las dos unidades es el encaminamiento dado al problema, relacionado a la forma en que cada servicio entiende la violencia como demanda inherente también a la esfera de salud.

Palabras clave: Atención primaria a la salud. Salud de la mujer. Violencia contra la mujer. Violencia doméstica. Violencia de género.

Recebido em 19/04/07. Aprovado em 04/06/08. 